\title{
Proton transfer during class-A GPCR activation: do the CWxP motif and the membrane potential act in concert?
}

\author{
Xuejun C. Zhang ${ }^{1,2}$, Ye Zhou ${ }^{1,2}$, Can Cao ${ }^{1}$ \\ ${ }^{1}$ National Laboratory of Biomacromolecules, CAS Center for Excellence in Biomacromolecules, Institute of \\ Biophysics, Chinese Academy of Sciences, Beijing 100101, China \\ ${ }^{2}$ University of Chinese Academy of Sciences, Beijing 100049, China
}

Received: 19 March 2018 / Accepted: 10 April 2018 / Published online: 12 June 2018

\section{INTRODUCTION}

G-protein-coupled receptors (GPCRs) form the largest receptor superfamily of eukaryotic cells (Flock et al. 2017). A typical GPCR protein contains seven transmembrane helices (TMs 1-7) (Palczewski et al. 2000). Upon activation, the GPCR opens a cavity on its cytoplasmic side, between TMs 5-6 and the remaining of the receptor, to interact with downstream effectors such as G-proteins and arrestins (Kang et al. 2015; Rasmussen et al. 2011). Based on phylogenetic analysis, GPCRs have been further categorized into several families. Among them, the rhodopsin-like class-A-referred to as such in the A-F classification system (Attwood and Findlay 1994; Kolakowski 1994) - is the largest family, including over 700 members from human genome alone. Class-A GPCRs (hereafter referred simply as "GPCRs") possess a number of highly conserved fingerprint motifs (Nygaard et al. 2009) which presumably play important functional roles in common signaling mechanisms shared by all members of their family.

While both the ligand binding on the extracellular side and interactions with effectors on the intracellular side have been extensively studied and their mechanisms are fairly well understood (Kang et al. 2015; Katritch et al. 2012; Rasmussen et al. 2011), the detailed mechanisms of transmembrane signaling remain under debate. Recently, we proposed the electrostatic transmembrane potential $(\Delta \Psi)$-driving hypothesis, a mechanism of agonistinduced, proton transfer-mediated activation for GPCRs (Zhang et al. 2014). This hypothesis is based on an

$\bowtie$ Correspondence: zhangc@ibp.ac.cn (X. C. Zhang) extensive range of biochemical studies, on structural data from decades of GPCR studies regarding the most conserved structural features of GPCRs, and on a long-time belief that these shared features are associated with a common activation mechanism for the entire class-A family (Schwartz and Rosenkilde 1996). Since GPCRs carry electric charges, usually non-uniformly distributed, activation of a GPCR is inevitably affected by the membrane potential (Barchad-Avitzur et al. 2016; Birk et al. 2015; Mahaut-Smith et al. 2008; Rinne et al. 2013; Sahlholm et al. 2008). More specifically, according to our hypothesis, activation of GPCR is associated with the redistribution of electric charges within the receptor. In the ground state of GPCR, a key proton-titratable residue, D2.50 (as per B-W numbering (Ballesteros and Weinstein 1995)) maintains a protonated state. This aspartate residue is $92 \%$ conserved in all GPCRs (where percentage conservation of a given position is estimated according to the online database GPCRdb (Isberg et al. 2016)). The electrostatic force exerted by $\Delta \Psi$ on the protonated D2.50 is balanced by hydrophobic mismatch forces as well as by an amphipathic helix-8 (H8) at the C-terminal end of TM7. This amphipathic helix is embedded in the intracellular surface of the membrane bilayer and links TMs 1, 2, and 7 through a hydrophobic core. Upon activation, agonist binding induces a rotation of TM3 relative to TM2. In turn, a conserved polar residue at the position 3.39 triggers deprotonation of D2.50. The released proton is driven by $\Delta \Psi$ and moves along a conserved proton wire. It enters a cavity, termed the middle cavity (or TM6 clamp (Hulme 2013)), and further protonates a water molecule trapped within. Interestingly, the protonated water ion is insulated within a hydrophobic environment. A group of 
conserved hydrophobic residues (including L/I/V-2.43, L2.46, L/I-3.43, I/L/M/V-3.46, L/V/I/A-6.37, and I/V/M/ L-6.40) form the middle cavity (Zhang et al. 2013), and their importance in GPCR activation is also unveiled by a recent structural survey (Venkatakrishnan et al. 2016). The protonated water ion $\left(\mathrm{H}_{3} \mathrm{O}^{+}\right)$is then again subjected to an electrostatic force from $\Delta \Psi$; this extra force cannot be balanced by H8, thus promoting a conformational change of the receptor required for the downstream signaling. In general, amphipathic helices are often located on the cytosolic side of the integral membrane proteins that undergo large conformational changes during their functional cycles, and constrain the terminal movement of associated TM helices to the cytosolic surface of the lipid bilayer (Zhang et al. 2018). In addition to the conserved H8, a class-A GPCR often contains a short amphipathic helix in the intracellular loop-2 region, probably playing an anchoring function similar to H8. Taken together, the putative activation process of class-A GPCRs is hereafter called the "D2.50 switch."

It is encouraging that the functions of many fingerprint motifs and conserved residues as well as of the amphipathic H8 of GPCRs can be explained with our newly proposed $\Delta \Psi$-driving hypothesis (Zhang et al. 2014). One exception, however, is a highly conserved motif in TM6, whose functional roles remain enigmatic. More specifically, TM6 contains a conserved CWxP motif (where " $\mathrm{x}$ " stands for any amino acid residue) (Nygaard et al. 2009). P6.50 (99\% conserved) of this motif generates a break in TM6 acting as a hinge, presumably facilitating the conformational change observed during GPCR activation. However, the functional roles of $\mathrm{C} 6.47$ and W6.48 remain controversial at best. Interestingly, Cys and Trp are the two types of amino acid residues showing the most extreme pattern of conservation in a statistical survey; namely, both are frequently located at positions either highly conserved or highly degenerated (i.e., poorly conserved) (Marino and Gladyshev 2010). This observation suggests that strong selective pressure exists on keeping Cys as well as Trp residues in crucially important locations (e.g., functional sites), whereas in other positions Cys and Trp residues are less likely to be conserved because of fewer codons. In the following, we will discuss the unique properties and putative functional roles of C6.47 and W6.48 in the framework of our $\Delta \Psi$-driving hypothesis.

\section{DEPROTONATION OF CYS6.47}

C6.47 is conserved in nearly $70 \%$ of all class-A GPCRs, with Ser being a distant second choice $(10 \%)$ for this position. Unlike the other conserved pair of Cys residues in the GPCR superfamily, which forms a characteristic disulfide bond on the extracellular side of the receptor, C6.47 is not involved in any disulfide bond formation.

For integral membrane proteins, any existing disulfide bonds are exclusively located on the extracellular side, as oxidative environments are only available in the lumen of the endoplasmic reticulum (ER) and in the extracellular space. In the transmembrane region, in contrast, Cys residues are not involved in disulfide bonds but are often exchangeable with Ser residues. Cys and Ser residues are similar in geometry and are considered of possessing similar chemical properties (Marino and Gladyshev 2010). On the one hand, Cys is classified as a polar residue based on its physical-chemical properties. On the other hand, Cys (non-disulfide bonded) residues are mostly found in buried positions and are therefore considered hydrophobic. This peculiar discrepancy suggests that if Cys residues are positioned inside a protein, it is likely due to functional reasons, even though this results in an energetic cost, as it negatively interferes with folding and stability. Why then does the position 6.47 predominantly favor Cys over Ser? One clear difference between Cys and Ser is their $\mathrm{p} K_{\mathrm{a}}$ values: Cys ( $\mathrm{p} K_{\mathrm{a}} 8.5$ in solution (Poole 2015)) is much easier to deprotonate than $\operatorname{Ser}\left(\mathrm{p} K_{\mathrm{a}} 14.2\right)$. In fact, the intrinsic $\mathrm{p} K_{\mathrm{a}}$ of a Cys residue is the closest to the physiological $\mathrm{pH}$ of all of the naturally occurring amino acids. Their $\mathrm{p} K_{\mathrm{a}}$ difference partially explains why Cys pairs are able to form disulfide bonds, while Ser residues never form similar covalent bonds: in disulfide bond formation, one of the Cys residues is deprotonated first, thus permitting a nucleophilic attack on the other Cys residue (Poole 2015). As the most strictly selected amino acid residue in evolution (Marino and Gladyshev 2010), cysteine is among the most frequently used residues in catalytic sites of enzymes, often activated by a neighboring general-base thus initiating a nucleophilic attack (Lu et al. 2017). Furthermore, analogous to an Asn (Gln) residue mimicking the protonation state of an Asp (Glu) residue, a Ser residue may mimic the protonation state of a Cys residue. Therefore, if a Cys residue is not exchangeable for a Ser residue at a given position in a protein, it is likely that the deprotonation-thus the change of the protonation status-of this Cys residue is essential for the function of the protein. Similar to an acidic residue, a Cys residue is more likely to be deprotonated when its micro-environment becomes electropositive (Awoonor-Williams and Rowley 2016). Under such conditions, the $\mathrm{p} K_{\mathrm{a}}$ value of the Cys residue can decrease to as low as 2.9. Therefore, the question arises as to whether $\mathrm{C6.47}$ switches its protonation status during GPCR activation.

C6.47 is strategically located on the cytoplasmic side of the helix kink at P6.50 and on the interface between TM6 and TM7. In the ground-state crystal structures of 
GPCRs (e.g., of $\alpha_{2 \mathrm{~A}}$ adrenergic receptor $\left(\alpha_{2 \mathrm{~A}} \mathrm{AR}\right) / 4 \mathrm{EIY}$ (Liu et al. 2012)), the S $\gamma$ atom of C6.47 is located close to the $\delta$-amide group (a hydrogen bond donor) of the N7.45 sidechain (with a distance of $\sim 3.8 \AA$ and a proper angle for hydrogen bonding). This hydrogen bonding encourages deprotonation of C6.47. Position 7.45 is conserved as a polar residue, mainly Asn (67\%), and belongs to the NSxxNPxxY motif in TM7 (Fredriksson et al. 2003). In contrast to the ground state, in the active state (e.g., in $\beta_{2}$ adrenergic receptor $\left(\beta_{2} \mathrm{AR}\right) /$ 3SN6 (Rasmussen et al. 2011)), C6.47 and N7.45 slightly move from each other (to a distance of $\sim 4.3 \AA$ and an unfavorable angle for hydrogen bonding). This separation allows C6.47 to become protonated, and it is accompanied by the TM6 movement away from the remaining transmembrane helix bundle. In addition, in the active state capable of binding with downstream effectors (e.g., in $\beta_{2} \mathrm{AR} / 3 \mathrm{SN} 6$ ), the TM6 helix becomes less regular and curved within the region located at the cytoplasmic side of the P6.50 kink, a change consistent with the observed outward movement (i.e., opening) of the intracellular end of TM6. This helix curving causes the hydrogen bonds of the helix backbone to become more distorted from those in an ideal $\alpha$-helix, in particular exposing the backbone carbonyl oxygen of the residue 6.43. As a consequence, this exposed oxygen atom forms a hydrogen bond with the sidechain thiol (sulfhydryl) group of C6.47. This time, C6.47 is most likely to contribute a hydrogen bond donor. Therefore, formation of this potential hydrogen bond favors protonation of C6.47. In summary, while the microenvironment of 66.47 promotes its deprotonation in the ground state, it promotes protonation in the active state.

In the context of our $\Delta \Psi$-driving hypothesis, such an alternation in protonation status favors conformational change during the activation transition (Zhang et al. 2014, 2015). More specifically, the protonated C6.47 is subjected to an electrostatic force exerted by $\Delta \Psi$ which points in the intracellular direction. Together with hydrophobic mismatch forces, the electrostatic force generates a mechanical torque and facilitates both the opening of the intracellular side of the GPCR protein and the formation of the binding site for downstream effectors. In this sense, any ligand that weakens the C6.47-N7.45 interaction between TM6 and TM7 in the ground state should promote protonation of C6.47, subsequently stabilizing the active state. Therefore, in addition to the D2.50 switch, C6.47 likely serves as an auxiliary activation switch in GPCRs (Fig. 1).

Within the electric field of $\Delta \Psi$, C6.47 is located on the positive side of the key acidic residue D2.50. Thus, hypothetically, C6.47 becomes deprotonated during establishment of the ground state probably by forming the hydrogen bond with N7.45, and the released proton moves along a proton path (via N7.45- $\mathrm{H}_{2} \mathrm{O}$ ) finally protonating D2.50. However, during GPCR activation, the proton is unable to move back from D2.50 to C6.47, because this process would be against the electric field of $\Delta \Psi$. Instead, another proton is likely to be recruited from the extracellular space, a process likely mediated by a water molecule located at the kink of P6.50 (see the 1.8- $\AA$ crystal structure of $\left.\alpha_{2 \mathrm{~A}} \mathrm{AR} / 4 \mathrm{EIY}\right)$. Furthermore, the proton released from D2.50 during activation moves to a water molecule trapped in the middle cavity before being released into the cytoplasm. Thus, one effective proton apparently moves from the extracellular space to the intracellular side of the GPCR during the activation process. In agreement with this argument, the gating current of the muscarinic acetylcholine receptor-2 (M2 AChR) was previously estimated to be 0.85 proton per GPCR molecule (Ben-Chaim et al. 2006).

In accordance with the new hypothesis on the functional role of $\mathrm{C6.47}$, mutational variants $\mathrm{C} 6.47 \mathrm{~T}$ in $\beta_{2} \mathrm{AR}$ (Shi et al. 2002) and C6.47S/R in thyrotropin hormone receptor (Biebermann et al. 2012) result in constitutive $G_{\alpha s}$ activation. These mutations are equivalent to protonation of Cys or carrying an extra positive change, thus stabilizing the active state in the presence of $\Delta \Psi$ according to our hypothesis. Similarly, mutations at C6.47 in cannabinoid receptors CB1 and CB2 modified both ligand recognition and receptor activation (Pei et al. 2008; Picone et al. 2005). It has been proposed that C6.47 participates in the rearrangement of the TM6 and TM7 interface during GPCR activation (Olivella et al. 2013). However, in this previous hypothesis, the C6.47N7.45 hydrogen bond was thought to be formed between the thiol group of $\mathrm{C} 6.47$ and the $\delta$-carbonyl group of the N7.45 sidechain, and no Cys deprotonation was postulated. Given that the resolutions of currently available GPCR structures are not sufficiently high to determine the atom types at the terminus of the N7.45 sidechain, it is possible that the thiolate group of C6.47 forms a hydrogen bond with the $\delta$-amide group of N7.45 sidechain (as proposed here). Furthermore, such a sidechain rotamer conformation of N7.45 is in agreement with the original assignment of the 1.8- $\AA$ resolution, ground-state crystal structure of $\alpha_{2 A} A R / 4 E I Y$.

In addition, C6.47 of $\beta_{2} \mathrm{AR}$ was found to be more reactive with some sulfhydryl-specific reagents in the active state than the ground state (Rasmussen et al. 1999). This observation also indicated that GPCR activation alters the micro-environment of the thiol group of C6.47. However, it is generally assumed that in solutions, deprotonated thiol groups are more chemically active. Therefore, the change of $\mathrm{C} 6.47$ reactivity appears to be opposite to what would be predicted by 

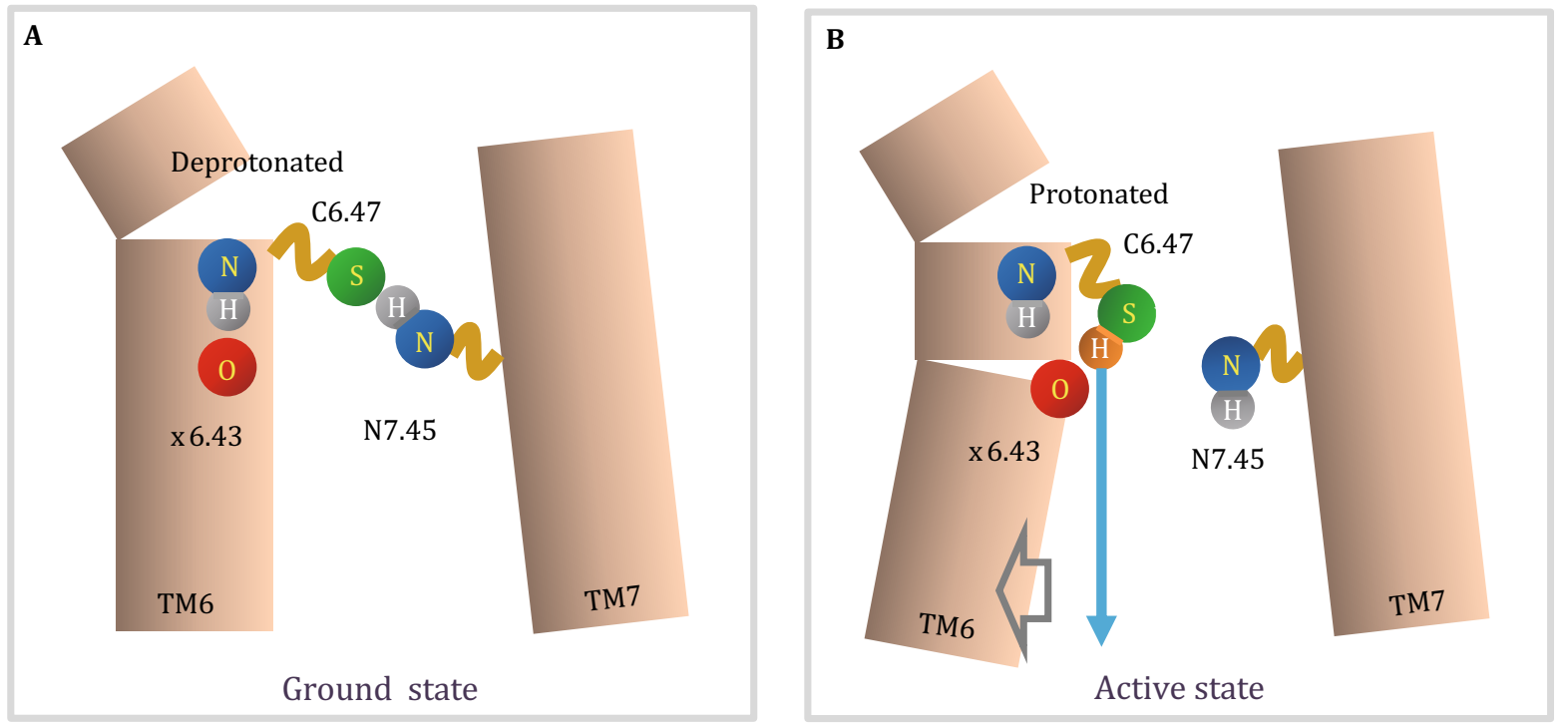

Fig. 1 Schematic diagram of Cys6.47 function. In the ground state (A), the N $\delta$ atom of N7.45 promotes deprotonation of the $\mathrm{S} \gamma$ atom of C6.47. The backbone 0 -atom of residue 6.43 and $\mathrm{N}$-atom of $\mathrm{C} 6.47$ form a standard $\alpha$-helix hydrogen bond. In the active state (B), TM6 and TM7 moves apart from each other (indicated with the open arrow), and more hydrogen bonds in TM6 become distorted than the ground state. Consequently, the $\mathrm{S} \gamma$ atom of $\mathrm{C} 6.47$ becomes protonated and forms a hydrogen bond with the backbone 0 -atom of residue 6.43 . The extra positive charge of the captured proton (golden sphere) is subjected to an electrostatic force (indicated with the blue arrow), which promotes and/or stabilizes the active state

our hypothesis. The underlying cause for this discrepancy may be the difference in reactivity of the thiol/ thiolate group, depending on whether it is surrounded by solvent phase or the hydrophobic environment inside a membrane protein. It has been argued that nucleophilicity of a Cys residue actually increases with $\mathrm{p} K_{\mathrm{a}}$ (Poole 2015), which is exactly what happens upon N7.45 moves away from C6.47. Future experimental studies (e.g., Raman spectroscopic analysis (Rothschild et al. 1993; Saint Clair et al. 2012)) on the C6.47 protonation status in both the ground and active states will help to verify the functional roles of C6.47.

\section{POLARIZATION OF TRP6.48}

At the position 6.48 within the CWxP motif of GPCRs, tryptophan is the dominant type of amino acid residue (68\% conserved), and phenylalanine comes as the second (16\%). W6.48 is in the middle of TM6, "vertically" located at the bottom of the ligand-binding pocket and above the conserved water-trapping "middle cavity". A recent NMR study suggests that W6.48 is involved in the same micro-switch as D2.50 (Eddy et al. 2018), probably through a shared hydrogen bond network (Liu et al. 2012). In addition, based on structures of the ground state, the sidechain rotamer of W6.48 was proposed earlier to assume a toggle role in GPCR activation (Shi et al. 2002). Surprisingly, however, W6.48 does not appear to change its sidechain rotamer in crystal structures of agonist-bound GPCRs reported later (Nygaard et al. 2009; Rasmussen et al. 2011; Rosenbaum et al. 2007). Furthermore, in many cases (for instance, GPR119, GPR39, $\beta_{2} \mathrm{AR}$, and the NK1 receptor), it was found that mutations of Ala substitution at W6.48 eliminate basal activities and strongly impair agonistinduced activation of downstream G-proteins (i.e., efficacy) without affecting agonist affinity (i.e., potency) (Holst et al. 2010). Thus, although it does not appear to be involved in a rotamer toggle switch induced by agonist binding, W6.48 is likely to function in a common activation mechanism. These observations raise the question as to what renders the Trp residue "irreplaceable" at the position 6.48. Is it possible that W6.48 also plays a functional role in the context of $\Delta \Psi$ ?

Statistically, integral membrane proteins contain more Trp residues than their soluble counterparts (Schiffer et al. 1992). Considering that only one genetic codon is available for tryptophan residues, the disproportionally abundant Trp residues likely play important roles in all classes of membrane proteins. The indole sidechain of Trp has an intrinsic electric dipole moment larger than 2 Debye, approximately pointing from the benzene ring to the pyrrole ring (Callis 1997). Since it contains nonlocalized electrons, the sidechain of a Trp residue can be further polarized within the indole plane by an external 
electric field, for example, of the transmembrane (or local) electrostatic potential. Accordingly, among all natural amino acid residues, tryptophan exhibits the highest polarizability. In other words, the indole moiety is characterized by a large dielectric constant along the direction of its long axis. Although their intrinsic dipoles are close to zero, sidechains of other aromatic amino acid residues may also possess similar but weaker induced dipoles. In general, the interactions between $\Delta \Psi$ and its embedded membrane protein can be divided into two parts: (1) the electrostatic forces on charged groups and (2) interactions of electric dipoles with the electric field of $\Delta \Psi$ (Hill 1985). The electrostatic force on each charged group is along the direction of the local electric field (i.e., electric line of force); the total electrostatic force is balanced by the forces of hydrophobic mismatch, keeping the protein molecule at an equilibrium position inside the lipid bilayer. Furthermore, if its dipole aligns with the electric field of $\Delta \Psi$ (corresponding to a low energy state), the Trp residue may stabilize the orientation of the membrane protein immerged inside the electric field of $\Delta \Psi$. The combination of both intrinsic and induced dipoles of Trp provides a possible explanation as to what the currently unknown function might be of the conserved W4.50 (94\%), whose sidechain is also aligned with the electric field of $\Delta \Psi$. In addition, the energy term of the interaction between an electric dipole and its surrounding electric field is the dot-product of the vectors of the dipole and field. Therefore, in a nonuniform electric field, an electric dipole prefers to move into a region of stronger strength of the electric field as well as to align with the electric line of force. As an example of such interactions, multiple Trp residues are often found to form a peripheral belt on the surface of the membrane protein near each of the two membranesolvent interfacial regions, where the electric field of $\Delta \Psi$ is strongly non-uniform, diminishing quickly away from the membrane. Such a Trp belt is likely to contribute to stabilization of the protein molecule in its proper position and orientation relative to the lipid bilayer. More generally, for a given $\Delta \Psi$, minimization of the total energy of the membrane protein-lipid bilayer complex system requires maximization of the effective dielectric constant(s) inside the membrane protein molecule, consequently increasing of the charge surface density on both sides of the protein exposed to the solvent. In other words, in the presence of $\Delta \Psi$, embedding polar groups, residues of high polarizability, and hydrogen bond networks inside the transmembrane region of the membrane protein is likely to be energetically favored for the overall stability of the protein-membrane complex system. Thus, the dipole of the indole sidechain of a Trp

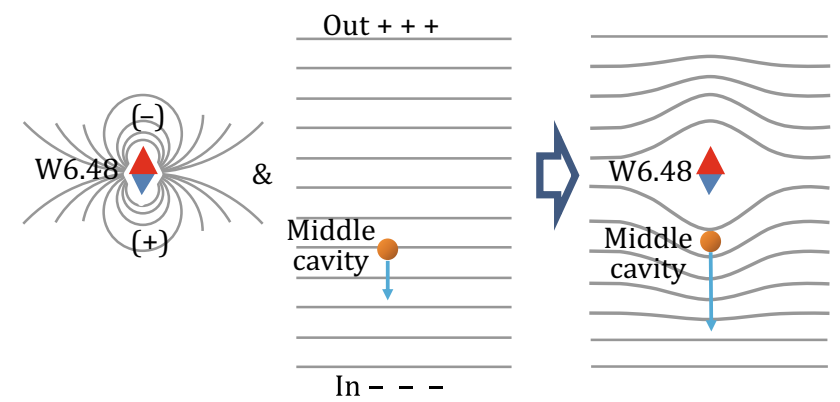

Fig. 2 Schematic diagram of W6.48 function. The electrostatic field of the dipole of the indole sidechain of W6.48 (depicted in diamond shape) is superimposed with an ideal uniform $\Delta \Psi$. Electric potentials are contoured as thin lines. In the middle cavity region, the combined electric field is stronger than that of $\Delta \Psi$ alone. Thus, the protonated water ion (golden sphere) is subjected to a stronger electric force (cyan line), which drives the conformational change of the activation process

residue may reshape the surrounding electric field, resulting in a locally "focused" electrostatic field, especially along the direction of the dipole (Fig. 2). The combined electric field from both the Trp dipole(s) and $\Delta \Psi$ further influences the properties of the host membrane protein.

These physical properties of a Trp residue may partially explain why W6.48 within the core of the 7-TM helix bundle is highly conserved across GPCRs. In reported crystal structures of GPCRs, the W6.48 sidechain is often "vertically" oriented such that both its intrinsic and induced dipoles are aligned with the electrostatic field of the negative-inside $\Delta \Psi$. Therefore, the combined dipole of W6.48 is likely to strengthen the electrostatic field underneath (i.e., in the cytoplasmic direction), where a few water molecules are trapped inside the middle pocket $(\sim 15 \AA$ distance from W6.48). In this pocket, the strength of the electric field from the W6.48 dipole is of about the same magnitude as that of $\Delta \Psi$ (see Box 1). As mentioned above, during activation of the GPCR, a proton released from D2.50 is captured by one of the trapped water molecules, and the strengthened electric field thus exerts an extra force to the protonated water ion $\left(\mathrm{H}_{3} \mathrm{O}^{+}\right)$, favoring the conformational change in the cytoplasmic region of the GPCR. In addition, the conserved F6.44 (76\%) is located between W6.48 and the middle pocket, presumably serving as an insulator to prevent electrical leakage across the receptor. In summary, both the ligand-binding cavity on the extracellular side and the G-protein-binding cavity on the cytoplasmic side result in focused electric field of $\Delta \Psi$ onto the middle transmembrane region of the receptor; W6.48 further enhances the electric field onto the conserved hydrophobic middle pocket, optimizing the process of proton transfer-mediated activation. 
Box 1 The electric field

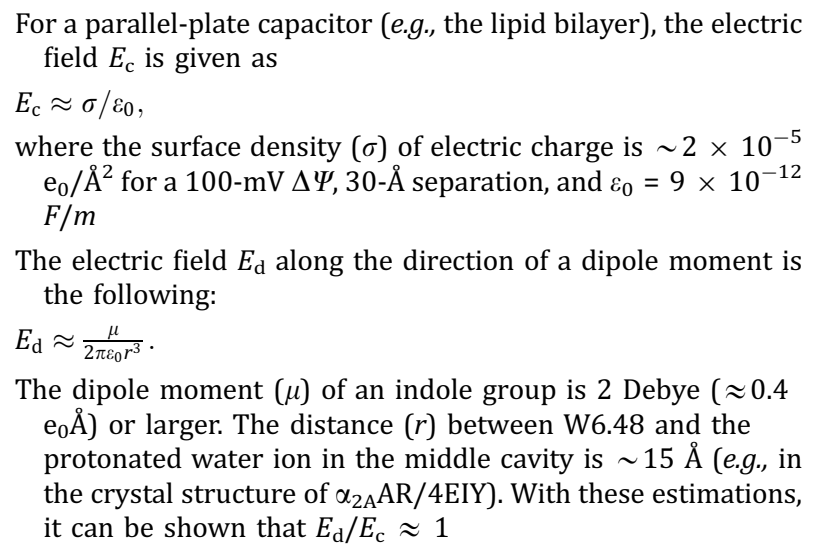

The electric field $E_{\mathrm{d}}$ along the direction of a dipole moment is the following:

$E_{\mathrm{d}} \approx \frac{\mu}{2 \pi \varepsilon_{0} r^{3}}$.

The dipole moment $(\mu)$ of an indole group is 2 Debye $(\approx 0.4$ $\left.\mathrm{e}_{0} \AA\right)$ or larger. The distance $(r)$ between W6.48 and the protonated water ion in the middle cavity is $\sim 15 \AA$ (e.g., in the crystal structure of $\left.\alpha_{2 A} A R / 4 E I Y\right)$. With these estimations, it can be shown that $E_{\mathrm{d}} / E_{\mathrm{c}} \approx 1$

To verify our hypothesis, it is essential to determine experimentally the extent to which W6.48 is polarized by $\Delta \Psi$, in combination with quantitative theoretical calculations. In addition, mutations at W6.48 may result in more compound effects on the buried hydrogen bond network formed around the D2.50 switch, and possibly on the C6.47 switch as well. Thus, interpretation of the data from mutagenesis studies requires cautions.

\section{SUMMARY}

Here we shed new light on the role of the electrostatic transmembrane potential in the function of membrane proteins. Based on current experimental evidence discussed, we updated our $\Delta \Psi$-driving hypothesis for class-A GPCR (Fig. 3). The effects of $\Delta \Psi$ on membraneembedded tryptophan and cysteine residues are likely to be of general importance for a more detailed understanding of the principles governing membrane protein function, and should attract attention from other fields studying the structures and molecular dynamics of membrane proteins.

\section{Abbreviations}

$\alpha_{2 \mathrm{~A}} \mathrm{AR} \quad \alpha_{2 \mathrm{~A}}$ adrenergic receptor

$\beta_{2} \mathrm{AR} \quad \beta_{2}$ adrenergic receptor

GPCR G-protein-coupled receptor

TM Transmembrane (helix)

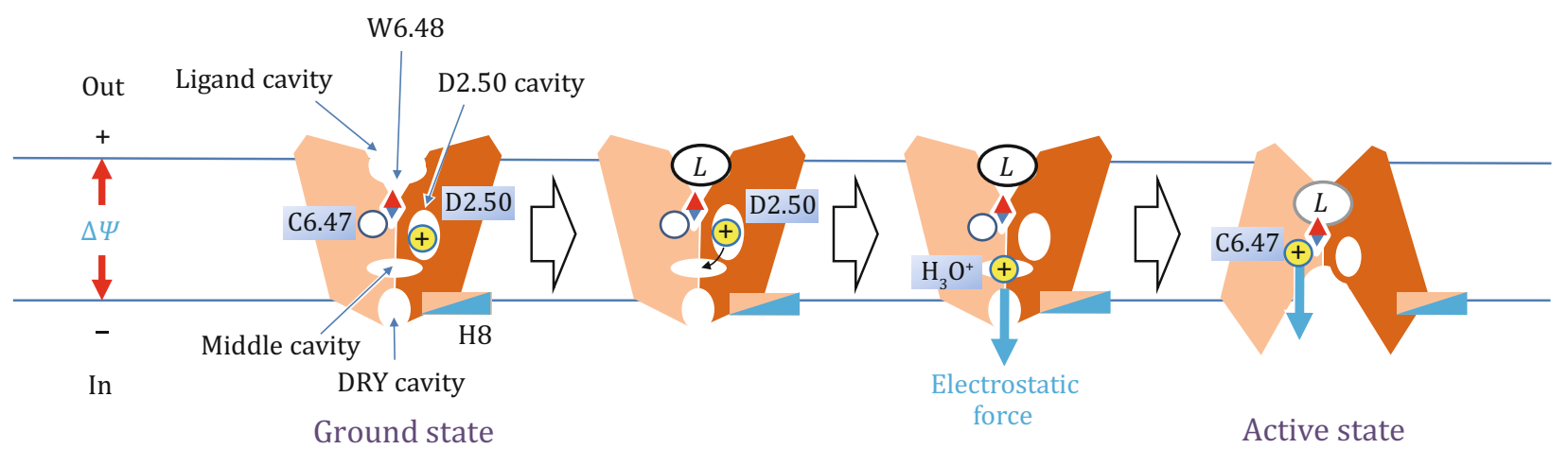

Fig. 3 Schematic diagram of activation mechanism of class-A GPCR. Updated mechanism based on the mechanism previously proposed in Zhang et al. (2014), with additional contributions from both C6.47 and W6.48. " $L$ " stands for a ligand (agonist) 
Acknowledgements The authors thank Dr. Torsten Juelich (Peking University, China) for linguistic assistance during the preparation of this manuscript. This work was supported by the Ministry of Science and Technology (China) (2015CB910104), the Chinese Academy of Sciences (XDB08020301), and National Natural Science Foundation of China (31470745).

\section{Compliance with Ethical Standards}

Conflict of interest Xuejun C. Zhang, Ye Zhou, and Can Cao declare that they have no conflict of interest.

Human and animal rights and informed consent This article does not contain any studies with human or animal subjects performed by any of the authors.

\section{References}

Attwood TK, Findlay JB (1994) Fingerprinting G-protein-coupled receptors. Protein Eng 7:195-203

Awoonor-Williams E, Rowley CN (2016) Evaluation of methods for the calculation of the $\mathrm{p} K_{\mathrm{a}}$ of cysteine residues in proteins. J Chem Theory Comput 12:4662-4673

Ballesteros JA, Weinstein H (1995) Integrated methods for the construction of three dimensional models and computational probing of structure-function relations in $\mathrm{G}$ protein-coupled receptors. Methods Neurosci 25:366-428

Barchad-Avitzur O, Priest MF, Dekel N, Bezanilla F, Parnas H, BenChaim Y (2016) A novel voltage sensor in the orthosteric binding site of the M2 muscarinic receptor. Biophys J 111:1396-1408

Ben-Chaim Y, Chanda B, Dascal N, Bezanilla F, Parnas I, Parnas H (2006) Movement of 'gating charge' is coupled to ligand binding in a G-protein-coupled receptor. Nature 444:106-109

Biebermann H, Winkler F, Handke D, Teichmann A, Gerling B, Cameron F, Eichhorst J, Gruters A, Wiesner B, Kuhnen P, Krude H, Kleinau G (2012) New pathogenic thyrotropin receptor mutations decipher differentiated activity switching at a conserved helix 6 motif of family A GPCR. J Clin Endocrinol Metab 97:E228-E232

Birk A, Rinne A, Bunemann M (2015) Membrane potential controls the efficacy of catecholamine-induced beta1-adrenoceptor activity. J Biol Chem 290:27311-27320

Callis PR (1997) 1La and 1Lb transitions of tryptophan: applications of theory and experimental observations to fluorescence of proteins. Methods Enzymol 278:113-150

Eddy MT, Lee MY, Gao ZG, White KL, Didenko T, Horst R, Audet M, Stanczak P, McClary KM, Han GW, Jacobson KA, Stevens RC, Wüthrich $\mathrm{K}$ (2018) Allosteric coupling of drug binding and intracellular signaling in the A2A adenosine receptor. Cell 172(68-80):e12

Flock T, Hauser AS, Lund N, Gloriam DE, Balaji S, Babu MM (2017) Selectivity determinants of GPCR-G-protein binding. Nature 545:317-322

Fredriksson R, Lagerstrom MC, Lundin LG, Schioth HB (2003) The G-protein-coupled receptors in the human genome form five main families. Phylogenetic analysis, paralogon groups, and fingerprints. Mol Pharmacol 63:1256-1272

Hill T (1985) Cooperativity theory in biochentistry: steady-state and equilibrium systems. Springer, New York

Holst B, Nygaard R, Valentin-Hansen L, Bach A, Engelstoft MS, Petersen PS, Frimurer TM, Schwartz TW (2010) A conserved aromatic lock for the tryptophan rotameric switch in TM-VI of seven-transmembrane receptors. J Biol Chem 285:3973-3985

Hulme EC (2013) GPCR activation: a mutagenic spotlight on crystal structures. Trends Pharmacol Sci 34:67-84

Isberg V, Mordalski S, Munk C, Rataj K, Harpsoe K, Hauser AS, Vroling B, Bojarski AJ, Vriend G, Gloriam DE (2016) GPCRdb: an information system for $G$ protein-coupled receptors. Nucleic Acids Res 44:D356-D364

Kang Y, Zhou XE, Gao X, He Y, Liu W, Ishchenko A, Barty A, White TA, Yefanov O, Han GW, Xu Q, de Waal PW, Ke J, Tan MHE, Zhang C, Moeller A, West GM, Pascal BD, Van Eps N, Caro N, Vishnivetskiy SA, Lee RJ, Suino-Powell KM, Gu X, Pal K, Ma J, Zhi X, Boutet S, Williams GJ, Messerschmidt M, Gati C, Zatsepin NA, Wang D, James D, Basu S, Roy-Chowdhury S, Conrad CE, Coe J, Liu H, Lisova S, Kupitz C, Grotjohann I, Fromme R, Jiang Y, Tan M, Yang H, Li J, Wang M, Zheng Z, Li D, Howe N, Zhao Y, Standfuss J, Diederichs K, Dong Y, Potter CS, Carragher B, Caffrey M, Jiang H, Chapman HN, Spence JCH, Fromme P, Weierstall U, Ernst OP, Katritch V, Gurevich VV, Griffin PR, Hubbell WL, Stevens RC, Cherezov V, Melcher K, Xu HE (2015) Crystal structure of rhodopsin bound to arrestin by femtosecond X-ray laser. Nature 523:561-567

Katritch V, Cherezov V, Stevens RC (2012) Diversity and modularity of $\mathrm{G}$ protein-coupled receptor structures. Trends Pharmacol Sci 33:17-27

Kolakowski LF Jr (1994) GCRDb: a G-protein-coupled receptor database. Recept Channels 2:1-7

Liu W, Chun E, Thompson AA, Chubukov P, Xu F, Katritch V, Han GW, Roth CB, Heitman LH, IJzerman AP, Cherezov V, Stevens RC (2012) Structural basis for allosteric regulation of GPCRs by sodium ions. Science 337:232-236

Lu GY, Xu YZ, Zhang K, Xiong Y, Li H, Cui L, Wang XP, Lou JZ, Zhai YJ, Sun F, Zhang XC (2017) Crystal structure of E. coli apolipoprotein N-acyl transferase. Nat Commun 8:15948

Mahaut-Smith MP, Martinez-Pinna J, Gurung IS (2008) A role for membrane potential in regulating GPCRs? Trends Pharmacol Sci 29:421-429

Marino SM, Gladyshev VN (2010) Cysteine function governs its conservation and degeneration and restricts its utilization on protein surfaces. J Mol Biol 404:902-916

Nygaard R, Frimurer TM, Holst B, Rosenkilde MM, Schwartz TW (2009) Ligand binding and micro-switches in 7TM receptor structures. Trends Pharmacol Sci 30:249-259

Olivella M, Caltabiano G, Cordomi A (2013) The role of Cysteine 6.47 in class A GPCRs. BMC Struct Biol 13:3

Palczewski K, Kumasaka T, Hori T, Behnke CA, Motoshima H, Fox BA, Le Trong I, Teller DC, Okada T, Stenkamp RE, Yamamoto M, Miyano M (2000) Crystal structure of rhodopsin: a G protein-coupled receptor. Science 289:739-745

Pei Y, Mercier RW, Anday JK, Thakur GA, Zvonok AM, Hurst D, Reggio PH, Janero DR, Makriyannis A (2008) Ligand-binding architecture of human CB2 cannabinoid receptor: evidence for receptor subtype-specific binding motif and modeling GPCR activation. Chem Biol 15:1207-1219

Picone RP, Khanolkar AD, Xu W, Ayotte LA, Thakur GA, Hurst DP, Abood ME, Reggio PH, Fournier DJ, Makriyannis A (2005) (-)-7'-Isothiocyanato-11-hydroxy-1', $1^{\prime}$-dimethylheptylhexahydrocannabinol (AM841), a high-affinity electrophilic ligand, interacts covalently with a cysteine in helix six and activates the CB1 cannabinoid receptor. Mol Pharmacol 68:1623-1635

Poole LB (2015) The basics of thiols and cysteines in redox biology and chemistry. Free Radic Biol Med 80:148-157

Rasmussen SG, Jensen AD, Liapakis G, Ghanouni P, Javitch JA, Gether U (1999) Mutation of a highly conserved aspartic acid in the beta2 adrenergic receptor: constitutive activation, 
structural instability, and conformational rearrangement of transmembrane segment 6. Mol Pharmacol 56:175-184

Rasmussen SG, DeVree BT, Zou Y, Kruse AC, Chung KY, Kobilka TS, Thian FS, Chae PS, Pardon E, Calinski D, Mathiesen JM, Shah STA, Lyons JA, Caffrey M, Gellman SH, Steyaert J, Skiniotis G, Weis WI, Sunahara RK, Kobilka BK (2011) Crystal structure of the beta2 adrenergic receptor-Gs protein complex. Nature 477:549-555

Rinne A, Birk A, Bunemann M (2013) Voltage regulates adrenergic receptor function. Proc Natl Acad Sci USA 110:1536-1541

Rosenbaum DM, Cherezov V, Hanson MA, Rasmussen SG, Thian FS, Kobilka TS, Choi HJ, Yao XJ, Weis WI, Stevens RC, Kobilka BK (2007) GPCR engineering yields high-resolution structural insights into beta2-adrenergic receptor function. Science 318:1266-1273

Rothschild KJ, Marti T, Sonar S, He YW, Rath P, Fischer W, Khorana HG (1993) Asp96 deprotonation and transmembrane alphahelical structural changes in bacteriorhodopsin. J Biol Chem 268:27046-27052

Sahlholm K, Marcellino D, Nilsson J, Fuxe K, Arhem P (2008) Voltage-sensitivity at the human dopamine D2S receptor is agonist-specific. Biochem Biophys Res Commun 377:1216-1221

Saint Clair EC, Ogren JI, Mamaev S, Russano D, Kralj JM, Rothschild KJ (2012) Near-IR resonance Raman spectroscopy of archaerhodopsin 3: effects of transmembrane potential. J Phys Chem B 116:14592-14601

Schiffer M, Chang CH, Stevens FJ (1992) The functions of tryptophan residues in membrane proteins. Protein Eng 5:213-214

Schwartz TW, Rosenkilde MM (1996) Is there a 'lock' for all agonist 'keys' in 7TM receptors? Trends Pharmacol Sci 17:213-216

Shi L, Liapakis G, Xu R, Guarnieri F, Ballesteros JA, Javitch JA (2002) Beta2 adrenergic receptor activation. Modulation of the proline kink in transmembrane 6 by a rotamer toggle switch. J Biol Chem 277:40989-40996

Venkatakrishnan AJ, Deupi X, Lebon G, Heydenreich FM, Flock T, Miljus T, Balaji S, Bouvier M, Veprintsev DB, Tate CG, Schertler GF, Babu MM (2016) Diverse activation pathways in class A GPCRs converge near the G-protein-coupling region. Nature 536:484-487

Zhang XC, Sun K, Zhang L, Li X, Cao C (2013) GPCR activation: protonation and membrane potential. Protein Cell 4:747-760

Zhang XC, Cao C, Zhou Y, Zhao Y (2014) Proton transfer-mediated GPCR activation. Protein Cell 6:12-17

Zhang XC, Zhou Y, Cao C (2015) Thermodynamics of GPCR activation. Biophys Rep 1:115-119

Zhang XC, Liu M, Lu G, Heng J (2018) Thermodynamic secrets of multidrug resistance: a new take on transport mechanisms of secondary active antiporters. Protein Sci 27(3):595-613 